\title{
Biological cold fusion
}

\section{Gottfried Schatz}

IN eukaryotic cells, the proteins of most membrane-bounded organelles are initially inserted into a progenitor organelle (the rough endoplasmic reticulum) and only later distributed to their different destinations. In this sorting process, proteins destined for transfer are sequestered within membrane vesicles that bud off from a donor organelle and then fuse with the appropriate acceptor organelle (see figure). Three papers by Rothman and collaborators on pages 355,397 and 398 of this issue ${ }^{1-3}$ show that vesicle fusion in several distinct branches of this complex distribution network requires the same cytosolic protein. This suggests that most, and perhaps all, of these fusions are driven by the same machinery which becomes activated once a vesicle has docked onto its specific target membrane.

Biochemical analysis of these transfer steps became possible after Rothman and his colleagues ${ }^{4}$ had worked out a cell-free system from Chinese hamster ovary (CHO) cells which catalyses the transfer of vesicles between different regions of the Golgi complex (see ref. 5 for a review). This transfer requires ATP, fatty acylCoA and at least two cytosolic proteins, one of which is inactivated by the sulphhydryl blocker $N$-ethylmaleimide (NEM). Subsequent work showed that the transfer involves several sequential steps which culminate in the fusion of vesicles with a medial Golgi sac; when the cell-free system is treated with NEM, vesicles bind to the target membrane but fail to fuse with it. Readdition of a cytosol fraction in the presence of ATP restores fusion. ATP and at least one NEM-sensitive cytosolic protein are thus necessary for the final fusion step.

Helped by this assay system, Rothman and his collaborators purified the NEMsensitive protein (which they call NSF), characterized it as a tetramer with subunits of relative molecular mass 76,000 , and raised a monoclonal antibody against it. This set the stage for the three studies reported in this issue.

The paper by Beckers et al. ${ }^{2}$ reports that NSF is also required for transferring vesicles between the rough endoplasmic reticulum and the Golgi complex. Transfer requires ATP and is inhibited by NEM or the monoclonal antibody against NSF. NSF is required in a late, calcium-dependent transfer step; this step is most likely the fusion step. However, the published data do not exclude the possibility that NSF is also needed at earlier steps.

Diaz et al. ${ }^{3}$ find that this versatile protein also participates in the fusion of endosomes in a cell-free system derived from macrophages. Again, involvement of the protein can be deduced from the sensitivity of the process to NEM or to the antiNSF monoclonal antibody, and from the finding that most of the inhibition by NEM is overcome by subsequent addition of NSF from CHO cells

To get a closer look at this protein, Wilson et al.' cloned and sequenced its gene and find that the deduced protein product has 48 per cent sequence similarity with the product of the SEC18 gene of
The sequence of mammalian NSF lacks any obvious hydrophobic regions resembling those of viral proteins which can induce membrane fusions by themselves. It is thus unclear whether NSF is a true 'fusogen' or whether it merely promotes the fusogenic action of some unknown partner protein. Whatever the answer, vesicle transport between membranes surely requires more than a single protein catalyst.

There is already evidence for a second cytosolic protein factor and for a membrane-associated NSF receptor, and the precise role of fatty acyl-CoA also remains to be clarified. Some of these questions will probably soon be answered as biochemical resolution of multicomponent systems is usually fairly rapid once the first component has been purified in an active state. This has now been achieved for the system discussed here.

In a eukaryotic cell, membrane vesicles that bud from a donor organelle and fuse with an acceptor organelle transfer membrane components and soluble vesicle contents between intracellular compartments, and between the inside and the outside of the cell. Membrane fusions now shown to be mediated by the NEMsensitive protein (termed NSF in mammals and SEC18 protein in yeast) are indicated by thick arrows. RER, rough endoplasmic reticulum; C, M and T, cis-, medial- and trans-Golgi compartments; TGN, trans-Golgi network; $E$, early endosome; PL, preysosome; S, secretory granule. (The scheme omits several pathwas not relevant to this discussion.)

yeast. This gene had already been shown by Schekman et al. ${ }^{6}$ to control the transfer of vesicles between the rough endoplasmic reticulum and the Golgi complex, and later studies suggested it has a function in endocytosis? ${ }^{7}$. The deduced sequence of the SEC18 protein and its biochemical features are typical of a cytosolic protein". Indeed, Wilson et al. show that a cytosol fraction from yeast can replace the endogenous NSF in the cell-free intra-Golgi transfer system from $\mathrm{CHO}$ cells; NSF activity is 10 times higher in a cytosol fraction from yeast cells that overproduce the SEC18 protein and undetectable in cytosol from cells bearing a mutant sec 18 allele. This last observation also suggests that, in yeast, SEC18 protein is the only functional NSF homologue.
The results from these new studies ${ }^{1.3}$ also raise the possibility that fusions between different organelles derived from the rough endoplasmic reticulum may all be catalysed by the same set of proteins. These proteins might merely act sequentially or as a multisubunit 'fusion machine' that is assembled at its site of action in response to as-yet unknown signals. Either of these mechanisms would resemble the action of complement proteins whose sequential activation and assembly is triggered by specific immune reaction on the target membrane. If cells use the same instrumentation for most or all of these fusions, the task of unravelling the molecular orchestration of membrane flow will be made much easier.

The papers by Rothman and collaborators in this issue are remarkable in several respects. First, they exploit an impressive array of clever in vitro assays. Second, they underscore the fact that a biological system can sometimes be simpler than originally surmised. Finally, they demonstrate the power of combining genetics with biochemistry, thereby providing yet another example of biological fusion.

Gottfried Schatz is in the Department of Biochemistry, Biozentrum, University of Basel, Klingelbergstrasse $70, \mathrm{CH}-4056$ Basel, Switzerland

1. Wilson, D. W et al. Nature 339. 355-359 (1989).

2. Beckers, C. J M et al Nature 339, 397-398 (1989).

3. Diaz, R. et al. Nature 339, 398-400 (1989).

4. Glick, B. S. \& Rothman, J. E. Nature 326, 309-312 (1987).

5. Schatz. G. Trends biochem. Sci. 10, 95. (1985).

6. Schekman, R. \& Novick, P. in The Molecular Biology of the Yeast Saccharomyces cerevisiae (eds Strathern, J. N., Jones, E. W. \& Broach, J. R.) 361-398 (Cold Spring Harbor Laboratory, New York, 1982

7. Riezman, H. Cell 40, 1001-1009 (1985)

8. Eakle, K. A. Bernstein. M. \& Emr. S. D. Molec. cell. Biol. 8. 4098-4109 (1988) 